\title{
The space-time cube as an effective way of representing and analysing the streetscape along a pedestrian route in an urban environment
}

\author{
Thomas Leduc ${ }^{1,}{ }^{*}$, Vincent Tourre ${ }^{2}$, and Myriam Servières ${ }^{2}$ \\ ${ }^{1}$ AAU-CRENAU, CNRS, École Nationale Supérieure d'Architecture de Nantes, France \\ ${ }^{2}$ AAU-CRENAU, École Centrale de Nantes, France
}

\begin{abstract}
The graphical representation of a complex polygonal object, such as an isovist field (i.e. a 2D isovist in its evolution over time along a pedestrian path in an urban environment) is a hard research issue that was stated in the late 1970s by Benedikt [1]. The generalized space-time cube from Bach and al. [2] is a conceptual framework allowing to propose innovative implementations for the analysis and the display of this complex object. The purpose of this article is to situate, within this formal framework, all the graphical representation solutions that have already been developed for isovist fields. It also aims to identify the problems raised by each of these solutions (spatial anchoring, arbitrary sampling, etc.) and to propose related solutions.
\end{abstract}

\section{Introduction and state of the art}

\subsection{Urban space analysis and visibility issues}

Because a strong trend at work today is to develop and design cities that allow people to adapt and mitigate phenomena related to major issues, such as climate change, several new criteria have emerged for the design of sustainable and healthy cities. These new criteria have to do with walkability, role of the streetscape in encouraging the development of pedestrian mobility, physical activity and well-being. As [3] points out, indicators related to walkability are not only the $\mathrm{D}$ variables (design, density, diversity, destination accessibility, or distance to transit) and must necessarily include "the microscale streetscape features that affect the pedestrian experience."

This attention to the landscape and its influence on pedestrian behaviour is part of a long line of work on the interactions between individuals and their built environment in terms of environmental psychology. It led to the identification of about fifty urban qualities likely to influence the behaviour of pedestrians moving in the street space [4].

\footnotetext{
* Corresponding author: thomas.leduc@crenau.archi.fr
} 
The literature on visibility studies extends this questioning and provides definitions and operating procedures; tools that make it possible to formalize and to measure the essence of places in terms of some of the involved urban design qualities. In the state of the art, which aims to "explore the spatial properties of the visual structure inherent in space" [5], we can highlight the isovist (Figure 1a) and isovist field (Figure 1b) as pioneering methodological tools proposed by [1]. The isovist is indeed a tool that easily answers the questions raised by [6]: "How far can we see, and how much can we see?". All key questions according to the author "in developing good design".

Even if other tools exist, such as the sky opening studied by [7], to analyse and even design urban space, we will focus in the current article only on isovists for their ability to synthesize, at any generation point, the immediately surrounding open space.
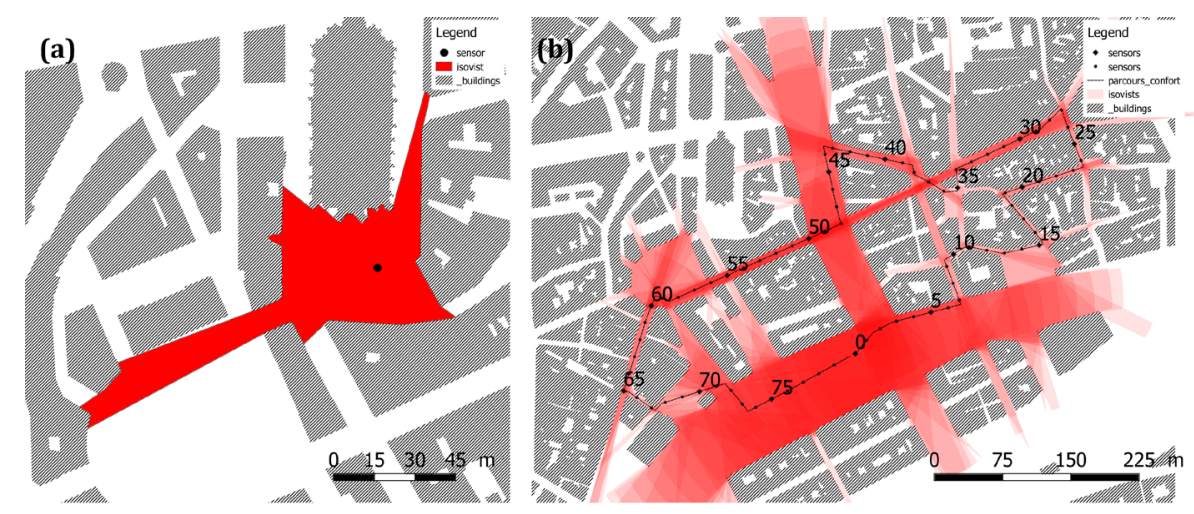

Fig. 1. (a) On the horizontal plane of the gaze, the isovist is the set of points seen from a given position (black disc); (b) The $1.6 \mathrm{~km}$ long pedestrian route is sampled every $20 \mathrm{~m}$. An isovist is then calculated at each sampling point. The corresponding isovist field is drawn in a flat representation. The darker the colour, the more the corresponding portion of urban space is seen along the path.

\subsection{Visibility representation issues along an urban route}

According to [1]'s definition, in an environment composed of opaque facades, the isovist associated with a given vantage point corresponds to all the points seen from the corresponding position in all directions of space. In the case of a 2D planar environment, such as the one presented in [1]'s pioneering article, the isovist is a bounded polygon representing a horizontal slice of the pedestrian's immediate surrounding space. In the absence of any opaque surface, it may be useful to limit the scope of the gaze with an artificial horizon. This location-specific visual shape holds the same characteristics as a "visual pattern" as defined by [8] and therefore information extracted from the isovist provides a synthetic view of the studied terrain.

With reference to the work on Gibson's motion perspective, [1] proposes "a cumulative understanding of the form of the environment [...] by perceiving variants and invariants in the transformation [...] caused by the observer's movement." Indeed, because "the view of and visual exposure to the environment will gradually and sometimes suddenly change with [the] position", the corresponding set of isovists and their shapes and sizes, but also the nature of their boundaries, vary along the way so as to create some sort of footprints' field specific to a given environment. To move beyond the study of single isovist and to provide an analytical tool to study a set of positions, he therefore proposes the isovist field. 
However, this cumulative visual experience exposes the designer to a difficulty in terms of representation, as can be seen in Figure 1b. The juxtaposition of polygons in the same horizontal plane causes overlaps. To overcome this difficulty and exhibit the structure of the set of surrounding visual spaces, [1] proposes two strategies. The first consists in moving a panoptic light source along the path in an analogue mock-up. All the illuminated points of the mock-up correspond to the $3 \mathrm{D}$ isovist associated with the position of the light source. The second, described as more analytical by its author, consists in building a Minkowski model. This model is a sequential stack of 2D isovists where the Z-axis embeds the time dimension. Finally, the author proposes to apply a dimensional reduction to this isovist field and to study scalar fields (the isovist is abstracted at any point by a measure value such as its area). This simplified representation model makes it possible to derive iso-potential lines in a traditional way.

\subsection{The space-time cube as a framework for temporal data visualization}

A pedestrian's route through the city is a complex object that has a temporal dimension in addition to traditional spatial dimensions. As such, it is time-geography insofar as time and space are fundamental dimensions for the analysis of its dynamics. [9] proposed innovative visual objects such as the space-time cube to illustrate in a graphic way this type of phenomenon. More recently, [2] has developed a conceptual framework for the visualization of temporal data based on generalized space-time cubes. According to [10], "maps are used to stimulate visual thinking about geospatial patterns, relationships, and trends." The question is therefore whether the space-time cube is a relevant geovisual solution for the analysis of complex data such as an isovist field.

In this article, after a presentation of the conceptual framework developed by [2] and a review of some of the elementary operations resulting from their study, we will show that the representation techniques already implemented in the field of visibility analysis using isovists, fit perfectly into this formalism. With the help of this formalism, we will then propose innovative visualizations before opening up to extended visualization challenges.

\section{Materials and method}

\subsection{Conceptual representation of spatial data based on the space-time cube}

According to [2], "a space-time object is a geometrical object within a space-time coordinate system". The latter is a three-dimensional Euclidean space that adds a temporal dimension to a two-dimensional geographical space. In such a space, it is very common to represent the trajectory of a moving point object in motion on a plane map background by a static 3D curve. However, in the specific case of the representation of an isovist field, it is a matter of displaying not a point trajectory, but a collection of polygons as stated before. The issue lies in adapting a process for visualizing the evolution of a dimensionless object over time, to the visualization of the evolution and superimposition of a $2 \mathrm{D}$ shape over time. Regarding the issue of the representation device, the space-time cube is by definition a $3 \mathrm{D}$ object and its $2 \mathrm{D}$ visualization is part of the $3 \mathrm{D}$ rendering problems (with occlusion, depth ambiguity, perspective distortion, colour distortion and navigation difficulties [2]). To bypass the edge effects of a projection of a space-time cube on a 2D support as computer screens, various techniques exist to reduce its complexity and dimensionality. 


\subsection{Review of some elementary operations related to the space-time cube}

According to [2], "the ultimate goal of space-time cube operations is to transform a spacetime cube into a space-time object whose shape is compatible with the shape of the media employed to convey the information". With this constraint, they then develop a taxonomy of elementary operations that they group into four categories: extraction (selecting a subset of the space-time object), flattening (dimensional reduction by aggregation), geometry transformation, and content transformation.

In the extraction category, we distinguish the so-called time cutting operation. This operation consists in extracting a $2 \mathrm{D}$ subset of the space-time object that corresponds to a single time cut (slice without thickness). In the flattening category, we can mention the time flattening which is a dimensional reduction operation by aggregating time. In the category of geometric transformations, we can mention the time scaling operation, which aims to resize the space-time object along the temporal axis (an acceleration of the phenomenon under study is similar to a contraction and a slowing down to a dilation). Finally, in the category of content transformations, we can mention the time colouring operation, which aims to alter the colours of each time slice according to time.

\subsection{Focus on compound operations formalism}

The inherent complexity of certain space-time phenomena justifies the application of several elementary operations, in sequence, to the data set to be displayed. To formalize these complex processes, [2] introduced the notion of compound operation. Like the usual composition of functions in mathematics, a compound operation is a mechanism to combine elementary operations to build composite ones. To develop this formalism, the authors introduced in particular the operator + which corresponds to the composition and the operator * which refers to a repetition (operation applied several times).

Discrete time flattening, which is an operation that aims to reduce the complexity of the space-time cube by aggregating all data independently of the time-related dimension, is then written as follows: (time cutting) $*+$ time flattening. It consists of starting with a first step of sampling the space-time cube as a function of time. Then to apply an aggregation operation on the resulting layers while preserving the spatial dimensions.

\section{Case Study}

\subsection{Presentation of the study site}

In order to test the few techniques for representing the above-mentioned complex geographical objects, we have identified an urban fabric that is emblematic of the city of Nantes (France). This $700 \mathrm{~m}$ by $500 \mathrm{~m}$ portion offers a diversity of urban morphologies, on a mainly pedestrian sector, flat ground of constant altimetry. To survey this urban fragment, we have chosen a $1.6 \mathrm{~km}$ pedestrian route (see Figure $1 \mathrm{~b}$ ) that winds its way from the medieval district in the east to a district of classical architecture dating back to the $18^{\text {th }}$ and $19^{\text {th }}$ centuries in the west. A research study conducted in 2010 on this same route [11] showed that its direction, if it is perfectly explained from a chronological point of view, is perfectible in terms of better visibility of heritage objects. For obvious reasons related to the constrained 
size of this article, the illustrations we present from now on are limited to a small portion of this path (from sampling point \#30 to sampling point \#40, see Figure 1b).

\subsection{Review of techniques already implemented for 2D visualization of isovists}

The isovist presented in Figure 1a is a flat representation of the space-time cube at a given time step. It results from a single elementary time cutting operation. The isovist field shown in Figure $1 \mathrm{~b}$ is a flat representation of the initial space-time cube. It is the result of a compound operation namely discrete time flattening described in section 2.3. To be more precise, this compound operation embeds a content transformation operation (translucent filter) in order to produce an alpha blending aggregation. The resulting compound operation is written: (time cutting + time colouring)* + time flattening. The same isovist field could have been shown with several isovists side-by-side as in [1], thus corresponding precisely to the discrete time juxtaposing compound operation. It is indeed a matter of extracting "multiple time slices and placing them side-by-side or on a grid" [2].

The Minkowski model mentioned in [1]'s article is a 3D rendering resulting from a compound operation applied to the space-time cube of isovist field: (time cutting + space shifting)* $+3 D$ Rendering. In case this sequential stack of 2D isovists is coloured, as in Figure 2, an additional content transformation is applied and the corresponding compound operation is equal to (time cutting + space shifting + time colouring) $*+$ time scaling $+3 D$ Rendering. The time scaling operation that precedes the final 3D rendering step aims to facilitate the overall visualization of the shape by contracting it (by a factor of 2 ).

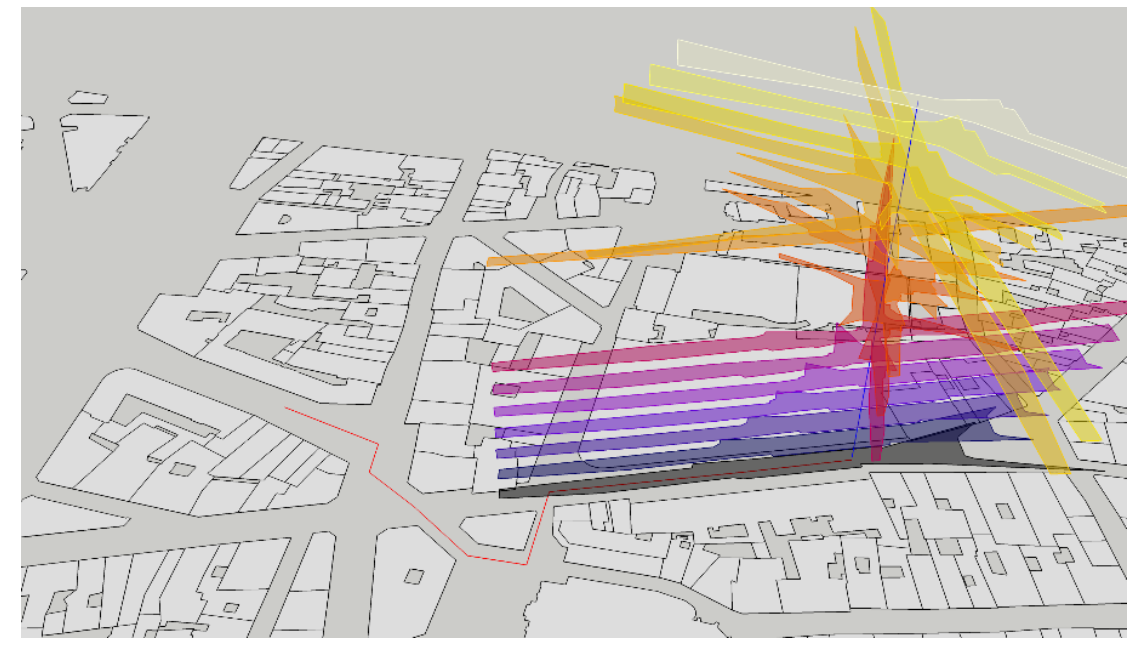

Fig. 2. Transposition of the "Minkowski model of the isovists along [the] given path" proposed by [1]. Sixteen isovists are stacked on the time axis, which is represented by the blue vertical axis. All the corresponding generation points are aligned on this axis. The more yellow the polygon is, the further away from the starting date.

The "Analog production of isovists along a path by point-source illumination of a model" stated in [1] is not a static visualization but a dynamic operation that consists of moving the cutting position over time. This is the compound operation known as animated time cutting according to the above-mentioned formalism. 


\subsection{Innovative 3D renderings}

The stack of isovists presented in Figure 2 has the advantage of providing a synthetic view of the evolution of the pedestrian's isovists during his walk in the city. Its formal qualities make it possible to highlight the bifurcations and configuration changes in the pedestrian's surroundings over time. However, the space shifting operation that systematically accompanies time cutting results in a loss of all anchor points because it translates all isovists into the same vertical.

To counteract this loss of anchoring and avoid getting lost on the background map, we propose to remove the space-shifting step in the aforementioned compound operation. The space-time object presented in Figure 3 is a $3 \mathrm{D}$ rendering resulting from the following compound operation applied to the generalized space-time cube: (time cutting + time colouring)* + time scaling $+3 D$ Rendering.

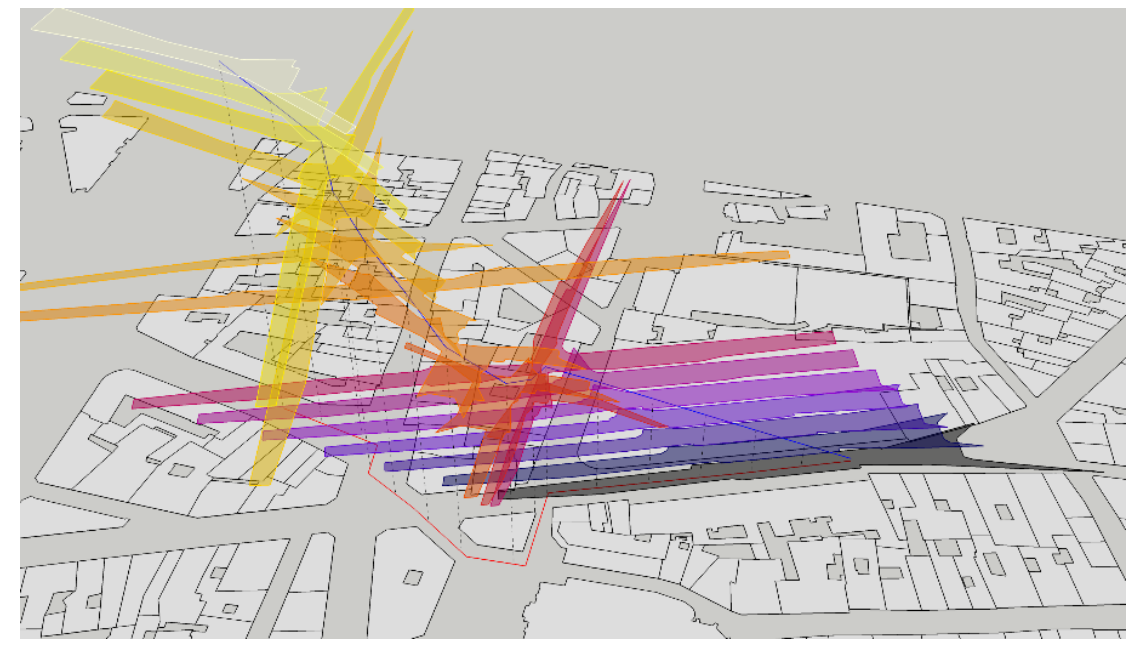

Fig. 3. By removing the space shifting operations in the Minkowski model from Figure 2, the isovists are relocated to match the path in spatial dimensions.

The shape presented in Figure 3 facilitates, through its stepped approach, a joint spatial and temporal interpretation of the isovist field. However, even if its sampling step is a little finer than that of Figure $1 \mathrm{~b}(12 \mathrm{~m}$ vs. $20 \mathrm{~m})$, one must recognize that this discretization remains coarse and that it may mask possible major visual events.

Figure 4 combines a response in terms of refinement of discretization, the sampling step is reduced to $2 \mathrm{~m}$, with a thickness representation of the corresponding "visual volume". Indeed, instead of a stack of isovist slices, we represent a global volume by connecting the contour points from one time step to the next time step. This rendering also has the effect of guaranteeing a form of continuity of the entire shape, that could be associated to a single $3 D$ Rendering operation, and thus allowing to show the raw shape of the isovist field. 


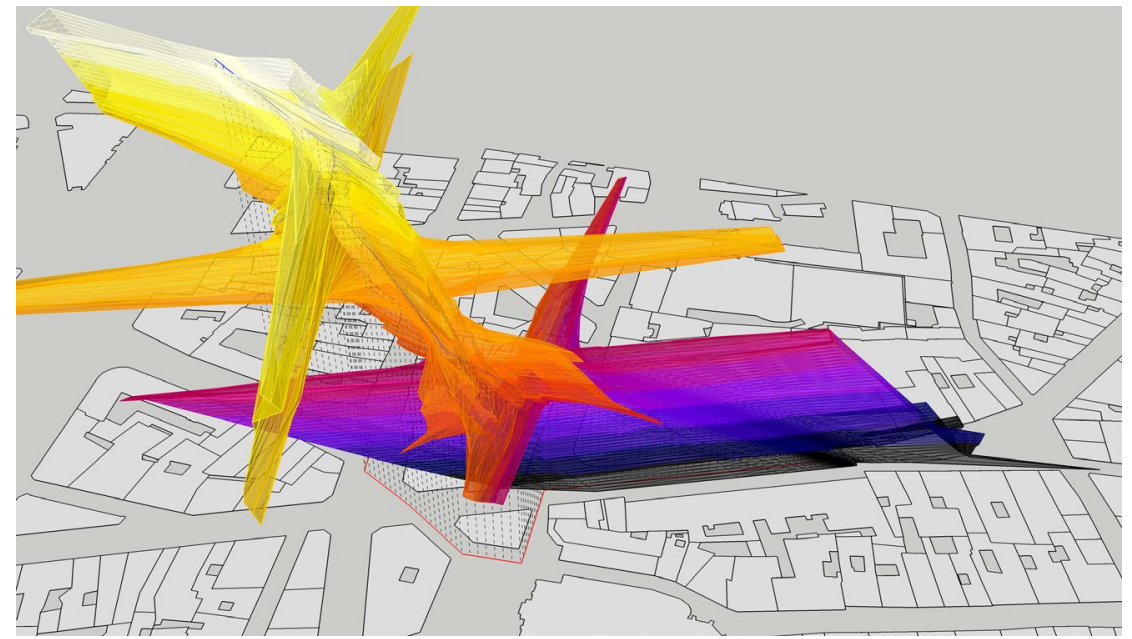

Fig. 4. Rendering in thickness of the space-time shape presented in Figure 3. In addition to refining the sampling step, the contour surfaces are drawn to shape a kind of "visual volume".

\section{Discussion}

\subsection{Immersion}

This article and the figures it embeds are intrinsically 2D objects. Being able to free ourselves from the 3D rendering step, by implementing stereographic rendering, would probably allow us a better understanding of an isovist field along a path (which is a typically $3 \mathrm{D}$ object). Indeed, by avoiding projection on a plane, we give free opportunity to immersion and navigation in the virtual space-time cube. We also allow to resize and adapt it to a human scale, as [12] points out: "It can be seen that the scanned object [...] is systematically enlarged by students within the virtual environment in order to reach a height at least equal to the size of the user. The model is now 'inclusive' and in turn operates a form of immersion that we will call expansive".

\subsection{Increase dimensionality}

As usual in the representation of the space-time cube, the background map in Figures 2, 3, and 4 is two-dimensional. To facilitate the location and identification of built volumes, we could imagine replacing this background plan with a three-dimensional urban model. However, this facility would have the disadvantage of introducing new masks and thus increasing occlusions. It would also introduce confusion in the use of the third dimension, which would be both spatial and temporal. In order to analyse the interstitial urban space in a volumetric perspective, we could imagine representing not two-dimensional isovists in their evolution over time but three-dimensional isovists. This dimensional increase of the item to be represented will consequently substantially increase the occlusion effects and depth ambiguities. 


\subsection{About adaptive sampling}

The sampling distances used in this study are arbitrary and systematic. However, there are portions of the route that present more or less visual events. It is therefore entirely legitimate, if not desirable, to consider adapting the sampling step to the regularity or irregularity of the course at each point. The partitioning of the urban space of [13], as well as its extension by [14], who proposes to qualify the space according to the importance of changes in visual surroundings, could be very useful in this regard.

\section{Conclusion}

In this article, we have stated that attention to the visual landscape surrounding pedestrians as they walk around the city is a useful operating concept in urban planning processes. To this end, [1] proposed the isovist field as a solution allowing a dynamic analysis (spatial and temporal) of the space covered. The representation of a complex polygonal object, such as a 2D isovist, in its evolution over time along a pedestrian path, is however a hard research issue. From the generalized space-time cube proposed by [2], which is a conceptual framework adapted to the analysis and representation of the isovist field, we have resituated all the representation solutions that have already been proposed. In this article, we have also identified the problems raised by each of these solutions and proposed innovative evolutions. We now have to validate the usability of our proposals through a set of controlled experiments.

\section{References}

1. M. L. Benedikt, "To take hold of space: isovists and isovist fields," Environ. Plan. B Plan. Des., vol. 6, no. 1, pp. 47-65, 1979

2. B. Bach, P. Dragicevic, D. Archambault, C. Hurter, and S. Carpendale, "A Descriptive Framework for Temporal Data Visualizations Based on Generalized Space-Time Cubes," Comput. Graph. Forum, vol. 36, no. 6, pp. 36-61, Sep. 2017

3. L. Yin, "Street level urban design qualities for walkability: Combining 2D and 3D GIS measures," Comput. Environ. Urban Syst., vol. 64, pp. 288-296, Jul. 2017

4. R. Ewing, S. Handy, R. C. Brownson, O. Clemente, and E. Winston, "Identifying and Measuring Urban Design Qualities Related to Walkability," J. Phys. Act. Heal., vol. 3, no. Suppl 1, pp. 223-240, 2006

5. M. Llobera, "Extending GIS-based visual analysis: the concept of visualscapes," Int. J. Geogr. Inf. Sci., vol. 17, no. 1, pp. 25-48, Jan. 2003

6. M. Batty, "Exploring isovist fields: space and shape in architectural and urban morphology," Environ. Plan. B Plan. Des., vol. 28, no. 1, pp. 123-150, 2001

7. J. Teller, "A spherical metric for the field-oriented analysis of complex urban open spaces," Environ. Plan. B Plan. Des., vol. 30, no. 3, pp. 339-356, 2003

8. S. Steiniger, "Enabling pattern-aware automated map generalization," University of Zürich, 2007

9. T. Hägerstrand, "What about people in Regional Science?," Pap. Reg. Sci. Assoc., vol. 24, no. 1, pp. 6-21, Dec. 1970

10. M. Kraak, "The Space-Time Cube Revisited from a Geovisualization Perspective," 
in ICC2003: Proceedings of the 21st international cartographic conference: cartographic renaissance, 2003, no. August, pp. 1988-1996

11. T. Leduc, F. Miguet, V. Tourre, and P. Woloszyn, "Towards a spatial semantics to analyze the visual dynamics of the pedestrian mobility in the urban fabric," in Lecture Notes in Geoinformation and Cartography, 2010

12. É. Bigot-Doll, "Échelles diffuses : Prospectives de conception via matière et VR," SHS Web Conf., vol. 47, p. 01002, Oct. 2018

13. J. Peponis, J. Wineman, M. Rashid, S. Hong Kim, and S. Bafna, "On the description of shape and spatial configuration inside buildings: convex partitions and their local properties," Environ. Plan. B Plan. Des., vol. 24, no. 5, pp. 761-781, 1997

14. T. Leduc, F. Miguet, and V. Tourre, "Motion Perspectives Integration in the Qualification of the Urban Spaces: Towards a 2D- and a 3D-Enrichment of the SPartition Method," in Urban Design and Representation, B. E. A. Piga and R. Salerno, Eds. Cham: Springer International Publishing, 2017, pp. 69-82 